\title{
Altered functional connectivity of the marginal division in migraine: a resting- state fMRI study
}

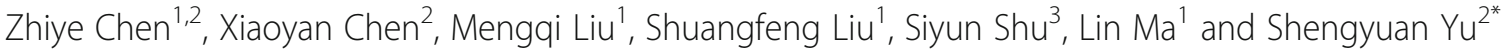

\begin{abstract}
Background: The marginal division of neostriatum $(\mathrm{MrD})$ is a flat, pan-shaped zone between the neostriatum and the globus pallidus, and previous documents demonstrated that it was involved in the modulation of pain. The aim of this study is to investigate the roles of the MrD of the human brain in the chronicization migraine using resting state functional magnetic resonance imaging (rs-fMRI).

Methods: Conventional MRI, 3D structure images, and rs-fMRI were performed in 18 patients with episodic migraines (EM), 16 patients with chronic migraine $(C M), 44$ patients with medication overuse headache plus chronic migraine $(\mathrm{MOH}+\mathrm{CM})$, and 32 normal controls $(\mathrm{NC})$. MrD was defined using manual delineation on structural images, and was selected as the seed to calculate the functional connectivity (FC).

Results: Compared with the NC group, the decreased FC of MrD was observed in the EM and CM groups, and increased FC of MrD was demonstrated in all patient groups. Compared with the EM group, the decreased FC of MrD was revealed in the $\mathrm{CM}$ and $\mathrm{MOH}+\mathrm{CM}$ groups, and the increased FC occurred only in the $\mathrm{CM}$ group. Increased FC of MrD alone was observed in the $\mathrm{MOH}+\mathrm{CM}$ group compared with that in the CM group.

Conclusion: This study confirmed the double neuromodulation network of $\mathrm{MrD}$ in pain modulation and migraine chronicization; however, the mechanism requires further investigation.
\end{abstract}

Keywords: Migraine, Marginal division of the neostriatum, fMRI, Functional connectivity

\section{Background}

The marginal division of neostriatum $(\mathrm{MrD})$ is a flat, pan-shaped zone between the neostriatum and the globus pallidus, consisting of spindle-shaped neurons and some special connections [1,2]. This area was first discovered at the caudal border of the striatum and the surrounding areas on the rostral edge of the globus pallidus in rat brains using histochemical techniques in 1988 [1]. Gradually more relevant papers were published, some of which demonstrated that this region was rich in neurotransmitters [3-5] and might also play an important role in learning and memory $[2,6-8]$.

In addition, neurophysiological studies showed that $\mathrm{MrD}$ was involved in the modulation of pain due to

\footnotetext{
*Correspondence: yusy1963@126.com

${ }^{2}$ Department of Neurology, Chinese PLA General Hospital, Beijing 100853, China

Full list of author information is available at the end of the article
}

nociceptive neurons localized exclusively in rat striatums $[9,10]$. Substance P, an important neuropeptide in the $\mathrm{MrD}$, plays a key role in learning and memory [11], which is also related to headache [12]. The rats with lesions of the MrD induced by kainic acid experienced altered perception of neuropathic pain behaviors. This may also be associated with the evident increased substance $\mathrm{P}$ in the ipsilateral spinal cord dorsal horn [13]. $\mathrm{Mu}$ opioid receptors (MORs) are localized in the MrD, and it is one member of the seven transmembrane family of G-protein coupled receptors, which may underlie pain and analgesia in the MrD of rat striatum [14]. To date, however, it has remained unknown whether and how MrD participates in pain modulation in the human brain in vivo.

Brain imaging and imaging analysis techniques can provide a possibility to explore and evaluate $\mathrm{MrD}$ in vivo in the human brain. A current study demonstrated that 
altered functional connectivity of $\mathrm{MrD}$ was shown in Alzheimer's disease by using resting state functional MRI (rs-fMRI) [15]. In our previous studies, this method was used to investigate the age and gender effects of functional connectivity of $\mathrm{MrD}$ for the normal subjects $[16,17]$. In clinical practice, the decreased functional connectivity of $\mathrm{MrD}$ was demonstrated in a patient with right extremity pain caused by a malacia lesion in the left putamen using rs-fMRI, which suggested that the MrD may contribute to the pain modulation [18].

The aim of this study is to investigate the roles of $\mathrm{MrD}$ in the chronicization of migraine using rs-fMRI. We hypothesized that $\mathrm{MrD}$ was involved in the pain modulation in headache, and that the decreased functional connectivity of $\mathrm{MrD}$ would generate the pain and might be the cause of migraine, while the increased functional connectivity of $\mathrm{MrD}$ would be complementary for pain and might compensate the dysfunction of MrD neuromodulation. To address this hypothesis, we obtained functional MR images for normal controls (NC), episodic migraine (EM) patients, chronic migraine (CM) patients, and medication overuse headache plus with chronic migraine $(\mathrm{MOH}+\mathrm{CM})$ patients. Firstly, the functional connectivity of $\mathrm{MrD}$ was performed with within-group analysis to explore the functional connectivity pattern of different subtypes of headache. Secondly, the between-group analysis was performed between the headache groups and the NC group to explore the varying functional connectivity in the different subtypes of headache. Lastly, between-groups analysis was performed among the different subtypes of headache to explore the altered pattern of functional connectivity.

\section{Method}

\section{Subjects}

One hundred and ten subjects were recruited, including 18 EM patients, $16 \mathrm{CM}$ patients, $44 \mathrm{MOH}+\mathrm{CM}$ patients, and 32 NCs. Patients were recruited from the International Headache Center, Department of Neurology, Chinese PLA General Hospital. All the following inclusion criteria should be fulfilled: 1) diagnosis of $1.3 \mathrm{CM}, 8.2$ $\mathrm{MOH}$, and 1.1 and 1.2 migraine based on the International Classification of Headache Disorders, third Edition (beta version) (ICHD-III beta) [19]; 2) no migraine preventive medication used in the past 3 months; 3 ) age between 20 and 60 years; 4) right-handed; 5) absence of any chronic disorders, including hypertension, hypercholesterolemia, diabetes mellitus, cardiovascular diseases, cerebrovascular disorders, neoplastic diseases, infectious diseases, connective tissue diseases, other subtypes of headache, chronic pain other than headache, severe anxiety or depression preceding the onset of headache, psychiatric diseases, etc.; 6) absence of alcohol, nicotine, or other substance abuse; and 7) patient's willingness to engage in the study. NCs were recruited from the hospital's staff and their relatives. Inclusion criteria were similar to those of patients, except for the first two items. NCs should never have had any primary headache disorders or other types of headache in the past year. General demographic and headache information were registered and evaluated in our headache database. Additionally, we evaluated anxiety, depression, and cognitive function of all the participants by using the Hamilton Anxiety Scale (HAMA) [20], the Hamilton Depression Scale (HAMD) [21], the Chinese version of Mini-Mental State Examination (MMSE), and the Montreal Cognitive Assessment (MoCA) Beijing Version (www.mocatest.org). The exclusion criteria were the following: cranium trauma, illness interfering with central nervous system function, psychotic disorder, and regular use of a psychoactive or hormone medication. The study protocol was approved by the Ethical Committee of Chinese PLA General Hospital and complied with the Declaration of Helsinki. Informed consent was obtained from all participants before the study. MRI scans were taken in the interictal stage at least 3 days after a migraine attack for EM patients. All the subjects were right-handed and underwent conventional MRI examination to exclude the subjects with cerebral infarction, malacia, or occupying lesions. Alcohol, nicotine, caffeine, and other substances were avoided for at least $12 \mathrm{~h}$ before MRI examination.

\section{MRI acquisition}

Images were acquired on a GE 3.0T MR system (DISCOVERY MR750, GE Healthcare, Milwaukee, WI, USA) and a conventional eight-channel quadrature head coil was used. All subjects were instructed to lie in a supine position, and formed padding was used to limit head movement. Conventional T2weighted image $(\mathrm{TR}=5000 \mathrm{~ms}$, $\mathrm{TE}=113.4 \mathrm{~ms}, \mathrm{FOV}=$ $24 \mathrm{~cm} \times 24 \mathrm{~cm}$, Matrix $=384 \times 384)$ and T1-FLAIR $(\mathrm{TR}=$ $2040 \mathrm{~ms}, \mathrm{TE}=6.9 \mathrm{~ms}$, FOV $=24 \mathrm{~cm} \times 24 \mathrm{~cm}$, Matrix $=$ $384 \times 256$ ) were obtained first. Then, the rs-fMRI was performed, during which subjects were instructed to relax, keep their eyes closed, stay awake, remain still, and clear their heads of all thoughts. Functional images were obtained using a gradient echo-planar imaging (EPI) sequence $(\mathrm{TR}=2000 \mathrm{~ms}, \mathrm{TE}=30 \mathrm{~ms}$, flip angle $=90$, slice thickness $=3 \mathrm{~mm}$, slice gap $=1 \mathrm{~mm}, \mathrm{FOV}=24 \mathrm{~cm} \times$ $24 \mathrm{~cm}$, Matrix $=64 \times 64$ ), and 180 continuous EPI functional volumes were acquired axially over $6 \mathrm{~min}$. Finally, a high resolution three-dimensional T1-weighted fast spoiled gradient recalled echo (3D T1-FSPGR) sequence was performed, which generated 360 contiguous axial slices $[$ TR (repetition time $)=6.3 \mathrm{~ms}$, TE (echo time $)=$ $2.8 \mathrm{~ms}$, flip angle $=15^{\circ}$, FOV (field of view) $=25.6 \mathrm{~cm} \times$ $25.6 \mathrm{~cm}$, Matrix $=256 \times 256$, slice thickness $=1 \mathrm{~mm}]$. None of the subjects complained of any discomfort or fell 
asleep during scanning. No obvious structural damage was observed based on the conventional MR images.

\section{Data processing}

All MR structural and functional images were processed using Statistical Parametric Mapping 8 (SPM8) (http:// www.fil.ion.ucl.ac.uk/spm) and the rs-fMRI data analysis toolkit (REST v1.8) [22] running under MATLAB 7.6 (The Mathworks, Natick, MA, USA).

The data preprocessing was carried out as follows: (1) The first ten volumes of each functional time course was discarded to allow for T1 equilibrium and the participants to adapt; (2) Slice timing; (3) Head motion correction; (4) Spatial normalization. These steps were performed by SPM8. No subjects had head motion with more than $1.5 \mathrm{~mm}$ displacement in $\mathrm{X}, \mathrm{Y}$, and $\mathrm{Z}$ direction or $1.5^{\circ}$ of any angular motion throughout the course of the scanning. The linear trend removal and temporal band-pass filtering $(0.01-0.08 \mathrm{~Hz})$ was performed by REST [22].

The functional connectivity analysis was performed as follows: (1) Spatial smooth (full width at half maximum $($ FWHM $)=6 \mathrm{~mm}$ ) using SPM8; (2) MrD was defined using manual delineation on a ch2bet template in MRIcron software (v6.6, www.mricro.com) (Fig. 1); (3) Functional connectivity computation of the left and right $\mathrm{MrD}$ were performed using REST (v1.8). The time

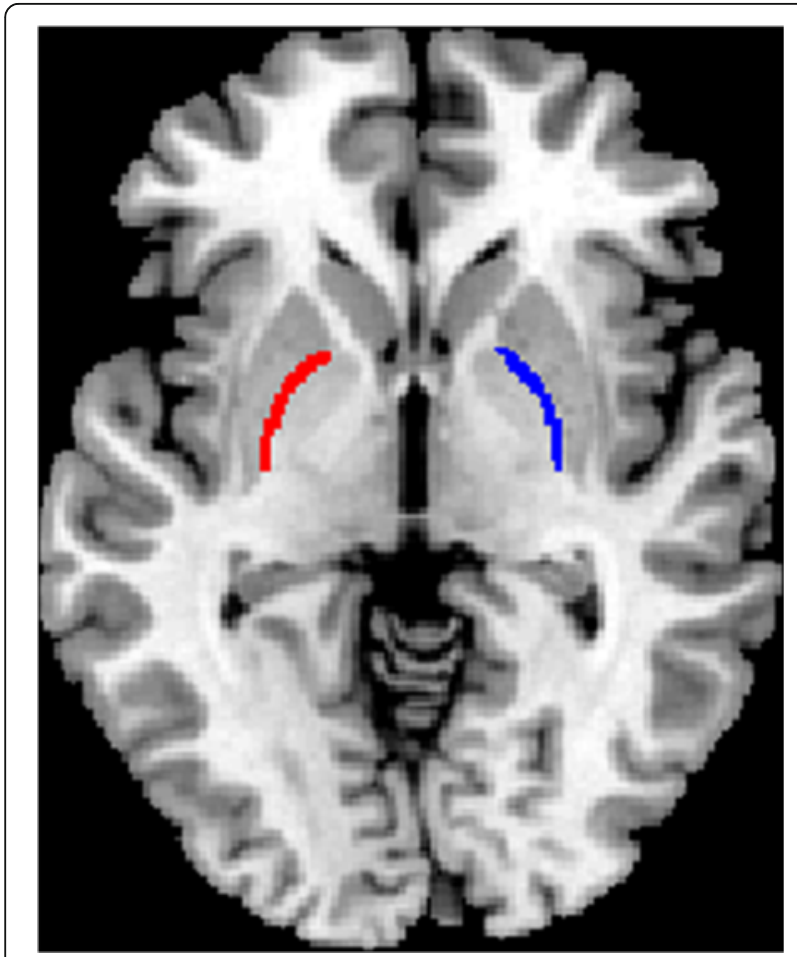

Fig. 1 Bilateral marginal divison was created by manual drawing based on ch2bet template in MRIcron software courses of bilateral MrD were extracted, and Pearson's correlations were used to calculate the functional connectivity between the extracted time courses and the averaged time courses of the whole brain in a voxel-wise manner. The white matter, cerebrospinal fluid (CSF), and the six head motion parameters were used as covariates. (4) The individual r-maps were normalized to Zmaps using Fisher's Z-transformation.

\section{Statistical analysis}

One-way analysis of variance (ANOVA) was applied to the comparison of the age, BMI, education, migraine duration, headache frequency, pain intensity, medication intake, HAMA, HAMD, MMSE, and MoCA score. An independent sample t-test was applied to the comparison of the duration of headache chronicity/medication between the $\mathrm{CM}$ and $\mathrm{MOH}+\mathrm{CM}$ groups. Significant difference was set at a $P$ value of $<0.05$. The statistical analysis was performed using SPSS 19.0.

One-sample t-tests were performed using the functional connectivity Z-maps to detect the regions with significant functional connectivity of $\mathrm{MrD}$. Analysis of covariance (ANCOVA) tests were performed to identify the regions with significant differences in connectivity to $\mathrm{MrD}$ among groups, covarying for age, gender, and education. Significance was set at a $P$ value of $<0.001$ without correction. The minimal number of contiguous voxels was set at 10 . The statistical analysis was performed by SPM 8 software.

\section{Results}

\section{Demography and neuropsychological test}

Demographic and clinical data are summarized in Table 1. One hundred and ten subjects were included in our study, comprising 44 patients with $\mathrm{MOH}+\mathrm{CM}$ (35 females, nine males, mean age $42.3 \pm 9.6$ years), 16 patients with CM (12 females, four males, mean age $42.4 \pm$ 8.7 years), 18 patients with EM (14 females, four males, mean age $37.8 \pm 7.9$ years), and $32 \mathrm{NCs}$ (24 females, eight males, mean age $41.3 \pm 10.8$ years). Although the age of the EM group tended to be lower than that of the other three groups, there was no significant difference for gender and age among groups. Body mass index (BMI) was significantly higher in the $\mathrm{CM}$ group than that of the EM and NC groups, and it was also higher in the $\mathrm{MOH}+\mathrm{CM}$ group than in the EM group, using ANCOVA analysis with age as the covariance. The education level of the $\mathrm{MOH}+\mathrm{CM}$ and $\mathrm{CM}$ groups was significantly lower than that of the EM and NC groups. We defined education in 6 levels from illiterate to master or higher as grades 1 to 6 .

The mean years of migraine was significantly higher in the $\mathrm{MOH}+\mathrm{CM}$ group (mean $17.8 \pm 9.1$ years) than that in the CM (11.3 \pm 9.3 years) and EM (12.4 \pm 8.1 years) groups. Headache frequency was significantly higher in 
Table 1 Demographic information of the subjects (mean \pm SD)

\begin{tabular}{|c|c|c|c|c|c|}
\hline & EM & $\mathrm{CM}$ & $\mathrm{MOH}+\mathrm{CM}$ & NC & $p$ value \\
\hline$N(F / M)$ & $18(14 / 4)$ & $16(12 / 4)$ & $44(35 / 9)$ & $32(24 / 8)$ & 0.97 \\
\hline Age (years) & $33.4 \pm 11.0^{* *}$ & $42.4 \pm 8.7$ & $42.3 \pm 9.6$ & $41.3 \pm 10.8$ & 0.01 \\
\hline Body mass index & $21.3 \pm 0.7$ & $24.4 \pm 0.7^{*}$ & $23.1 \pm 0.4$ & $22.5 \pm 0.5$ & 0.01 \\
\hline Education & $4.4 \pm 1.1$ & $3.4 \pm 1.3^{* *}$ & $3.1 \pm 1.2^{* *}$ & $4.7 \pm 1.0$ & 0.00 \\
\hline Migraine duration (years) & $12.4 \pm 8.1$ & $11.3 \pm 9.3$ & $17.8 \pm 9.1^{*}$ & - & 0.02 \\
\hline Headache frequency (days/month) & $3.5 \pm 2.7$ & $25.1 \pm 5.9^{* *}$ & $26.5 \pm 5.0^{* *}$ & - & 0.00 \\
\hline Pain intensity (VAS) & $8.3 \pm 1.5$ & $7.9 \pm 1.5$ & $8.0 \pm 1.4$ & - & 0.46 \\
\hline Duration of headache chronicity/medication overuse (years) & - & $3.0 \pm 3.3$ & $4.7 \pm 4.8$ & - & 0.20 \\
\hline Medication intake & $4.9 \pm 3.7$ & $4.3 \pm 3.8$ & $119.7 \pm 111.6^{* *}$ & - & 0.00 \\
\hline HAMA & $15.7 \pm 9.9^{* *}$ & $21.6 \pm 10.9^{* *}$ & $18.5 \pm 8.7^{* *}$ & $2.4 \pm 1.5$ & 0.00 \\
\hline HAMD & $10.9 \pm 7.3^{* *}$ & $16.3 \pm 10.5^{* *}$ & $19.9 \pm 11.9^{* *}$ & $1.1 \pm 0.9$ & 0.00 \\
\hline MMSE (adjusted by education) & $28.5 \pm 0.7$ & $26.9 \pm 0.7$ & $27.1 \pm 0.5$ & $28.3 \pm 0.6$ & 0.22 \\
\hline MoCA (adjusted by education) & $26.1 \pm 0.8$ & $23.5 \pm 0.9^{* *}$ & $24.8 \pm 0.6^{*}$ & $26.9 \pm 0.7$ & 0.02 \\
\hline
\end{tabular}

* VAS visual anologue score, HAMA Hamilton anxiety scale, HAMD Hamilton depression scale, MMSE mini-mental state examination, MoCA the Montreal cognitive assessment, $E M$ episodic migraine, $C M$ chronic migraine, $M O H+C M$ medication overuse headache plus with chronic migraine ${ }^{*}:$ compared to $N C s$ S $<0.05$; ${ }^{* *}$ :compared to NCs $P<0.01$

the $\mathrm{MOH}+\mathrm{CM}$ (mean headache days per month $26.5 \pm$ 5.0) and CM (25.1 \pm 5.9$)$ groups than that in the EM group $(3.5 \pm 2.7)$. There was no significant difference in chronic headache duration between the $\mathrm{MOH}+\mathrm{CM}$ group and the $\mathrm{CM}$ group or in pain intensity between the patient groups. The $\mathrm{MOH}+\mathrm{CM}$ group took much more medication (mean number of pills per month $119.7 \pm 111.6)$ than the CM $(4.3 \pm 3.8)$ and EM $(4.9 \pm 3.7)$ groups. The types of overused medication by $\mathrm{MOH}+$ CM patients included simple analgesics (3/44), triptan $(1 / 44)$, opioids (1/44), combination analgesics (33/44), and multiple drug classes (6/44). CM patients and EM patients most frequently took combination analgesics as painkiller. None of the migraine patients regularly took preventive medication during the past 3 months.

Anxiety and depression scores were significantly higher in the three headache groups than that in NC group. The $\mathrm{MOH}+\mathrm{CM}$ group showed a higher depression score, and the $\mathrm{CM}$ group showed a higher anxiety score than the EM group $(P<0.05)$. Cognitive function showed no significant difference among groups evaluated by MMSE but declined in the $\mathrm{MOH}+\mathrm{CM}$ (mean score $24.8 \pm 0.6)$ and $\mathrm{CM}(23.5 \pm 0.9)$ groups compared with the NC group when evaluated by MoCA.

\section{Functional connectivity of MrD - within-group analysis}

Within-group analysis was performed, and a false discovery rate (FDR) was used with a $p$ value set at $<0.05$ with an extended threshold of 10 voxels. Regions with connectivity to $\mathrm{MrD}$ in each of the groups were acquired, and the functional connectivity maps were marked on the SPM8 T1 template.
Regions with positive functional connectivity of $\mathrm{MrD}$ were mainly in the bilateral basal ganglion regions, thalamus, insula, hippocampus, and right medial frontal orbital cortex, and the regions of negative functional connectivity of $\mathrm{MrD}$ were in the bilateral temporal lobes and middle frontal lobes in the NC group (Fig. 2).

In the EM group, regions with positive functional connectivity were mainly located in bilateral basal ganglion regions, and no evident negative functional connectivity was observed (Fig. 2).

In the $\mathrm{CM}$ and $\mathrm{MOH}+\mathrm{CM}$ groups, regions with positive functional connectivity were located in bilateral basal ganglion regions. The regions with negative functional connectivity in the $\mathrm{MOH}+\mathrm{CM}$ group were larger compared with the CM group, which were located in the bilateral middle frontal gyrus, cingulum, and right temporal lobes (Fig. 2).

\section{Comparison of functional connectivity of MrD between the migraine groups and NC group}

Table 2 shows the altered functional connectivity of $\mathrm{MrD}$ in migraineurs compared with NCs. In the EM group, the brain regions with decreased functional connectivity were mainly located in the right insula, and the brain regions with increased functional connectivity were mainly located in the right precentral gyrus and anterior cingulate cortex (ACC) (Fig. 3). In the CM group, the decreased functional connectivity of $\mathrm{MrD}$ was observed in the right cuneus and left middle cingulum cortex (MCC), and the increased functional connectivity was detected in the bilateral middle frontal gyrus, left hippocampus, and middle temporal gyrus (Fig. 3). 


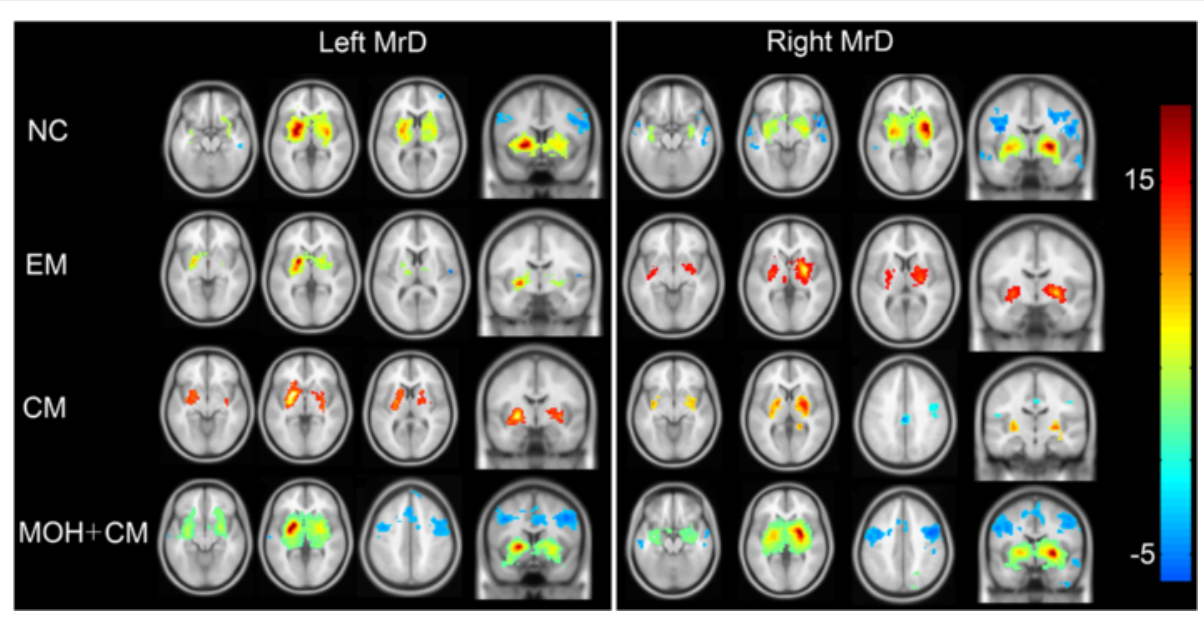

Fig. $2 \mathrm{MrD}$ functional connectivity averaged over subject in the brain. Warm and cool colors represent positive and negative correlations. NC, normal control; $\mathrm{EM}$, episodic migraine; $\mathrm{CM}$, chronic migraine; $\mathrm{MOH}+\mathrm{CM}$, medication overuse headache plus chronic migraine; L, left MrD; $\mathrm{R}$, right MrD

Interestingly, the decreased functional connectivity of $\mathrm{MrD}$ could not be observed in the $\mathrm{MOH}+\mathrm{CM}$ group, while the increased functional connectivity was demonstrated in the left parahippocampus, right middle frontal gyrus, and inferior temporal gyrus (Fig. 3).

\section{Comparison of functional connectivity of $\mathrm{MrD}$ among the} $\mathrm{CM}, \mathrm{MOH}+\mathrm{CM}$, and $\mathrm{EM}$ groups

Table 3 presents the altered functional connectivity of $\mathrm{MrD}$ among the $\mathrm{CM}, \mathrm{MOH}+\mathrm{CM}$, and $\mathrm{EM}$ groups. Decreased functional connectivity of $\mathrm{MrD}$ was detected

Table 2 Comparison of functional connectivity of MrD between headache group and HC group

\begin{tabular}{|c|c|c|c|c|c|c|c|}
\hline Group & Brain region & $k$ value & $P$ value & T value & $x$ & $y$ & z \\
\hline \multicolumn{8}{|l|}{ EM vs. NC } \\
\hline \multicolumn{8}{|l|}{$\mathrm{EM}<\mathrm{NC}$} \\
\hline Left & Insula_R & 27 & 0.000 & 4.2 & 36 & 21 & 6 \\
\hline Right & Insula_R & 23 & 0.000 & 4.3 & 33 & 18 & 6 \\
\hline \multicolumn{8}{|l|}{$\mathrm{EM}>\mathrm{NC}$} \\
\hline Left & Precentral_R & 13 & 0.000 & 4.5 & 42 & -15 & 36 \\
\hline Right & Cingulum_Ant_R & 17 & 0.000 & 4.4 & 12 & 45 & 21 \\
\hline \multicolumn{8}{|l|}{ CM vs. NC } \\
\hline \multicolumn{8}{|l|}{$\mathrm{CM}<\mathrm{NC}$} \\
\hline Left & Cuneus_R & 10 & 0.000 & 4.7 & 9 & -90 & 30 \\
\hline Right & Cingulum_Mid_L & 66 & 0.000 & 5.7 & 0 & -27 & 39 \\
\hline \multicolumn{8}{|l|}{$\mathrm{CM}>\mathrm{NC}$} \\
\hline \multirow[t]{3}{*}{ Left } & Frontal_Mid_L & 21 & 0.000 & 4.52 & -45 & 15 & 45 \\
\hline & Hippocampus_L & 12 & 0.000 & 4.06 & -30 & -39 & 0 \\
\hline & Frontal_Mid_R & 51 & 0.000 & 4.05 & 48 & 24 & 42 \\
\hline Right & Temporal_Mid_L & 15 & 0.000 & 4.35 & -54 & 3 & -21 \\
\hline \multicolumn{8}{|c|}{$\mathrm{MOH}+\mathrm{CMvs} . \mathrm{NC}$} \\
\hline \multicolumn{8}{|c|}{$\mathrm{MOH}+\mathrm{CM}>\mathrm{NC}$} \\
\hline Left & Frontal_Mid_R & 10 & 0.000 & 3.87 & 48 & 30 & 39 \\
\hline \multirow[t]{2}{*}{ Right } & Temporal_inf_R & 12 & 0.000 & 4.56 & 48 & -39 & -21 \\
\hline & ParaHippocampa_L & 18 & 0.000 & 4.42 & -18 & -21 & -24 \\
\hline
\end{tabular}

$R$ right hemisphere, $L$ left hemisphere, Ant anterior, Mid middle 


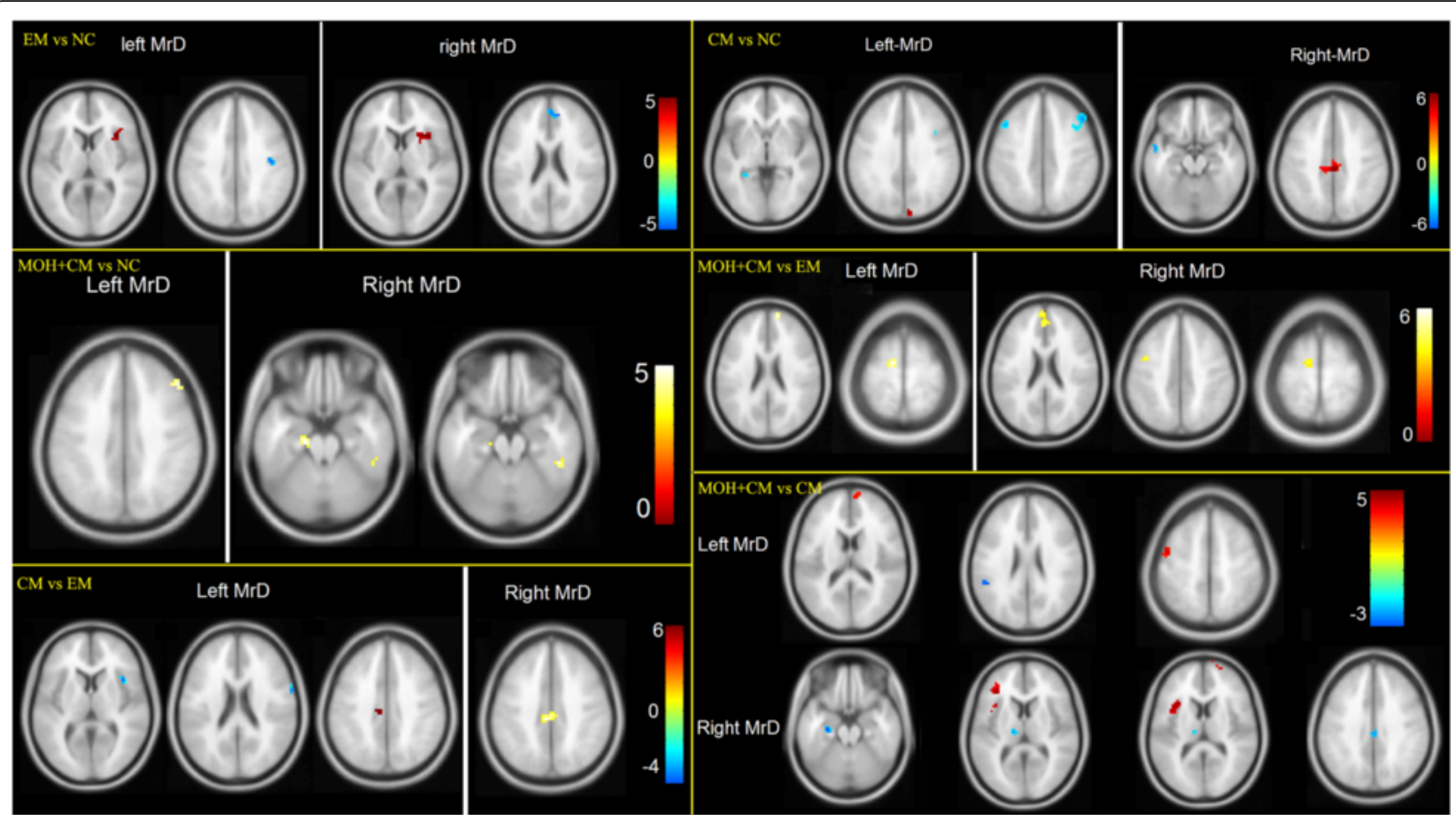

Fig. 3 Comparison of MrD functional connectivity among subjects group. Warm color represents decreased MrD functional connectivity in EM compared with NC, and cool color represent increased MrD functional connectivity. NC, normal control; EM, episodic migraine; CM, chronic migraine; $\mathrm{MOH}+\mathrm{CM}$, medication overuse headache plus chronic migraine; MrD, marginal division

in the left middle cingulum, and increased functional connectivity was observed in the right insula and precentral gyrus in the CM group compared with the EM group (Fig. 3).

In the $\mathrm{MOH}+\mathrm{CM}$ group, decreased functional connectivity of $\mathrm{MrD}$ was demonstrated in the bilateral medial superior frontal gyrus, left precentral gyrus, and supplementary motor area compared with the EM group (Fig. 3). No increased functional connectivity of $\mathrm{MrD}$ was observed.

The decreased functional connectivity of $\mathrm{MrD}$ was detected in the left precentral gyrus, the triangular part of the inferior frontal gyrus, the insula, the right medial superior frontal gyrus, and the superior frontal gyrus, and increased functional connectivity was observed in the left superior temporal gyrus, hippocampus, thalamus, and right middle cingulum in the $\mathrm{MOH}+\mathrm{CM}$ group compared with the CM group (Fig. 3, 4).

\section{Discussion}

Migraines are a common type of primary headaches with a reported prevalence of approximately $5.7 \%$ in men and $17.0 \%$ in women [23]. In China, the prevalence of migraine is $9.3 \%$ of the general population [24]. Migraines are also a major cause of chronic headaches, with approximately $2.5 \%$ of EM transformed to newonset CM [25]. The prevalence rate of CM is 2 to $4 \%$ of the general population [25], and that of $\mathrm{MOH}$ is 1 to $4 \%$ of the general population [26]. Therefore, chronicization of migraine is a worthy topic of further investigation.

The brain regions related with pain processing and modulation mainly included the prefrontal cortex, basal ganglia, thalamus, cingulate cortex, insula, cerebellum [27], and periaqueductal gray matter [28]. In this study, $\mathrm{MrD}$ was investigated to reveal its key roles in migraine chronicization using rs-fMRI.

Functional connectivity is actually the correlation analysis between the brain regions with $\mathrm{MrD}$ over the whole brain. The normal brain structure includes positive and negative functional connectivity to maintain the brain's functional balance. Altered functional connectivity may indicate the intrinsic pathophysiological changes for different brain disorders.

In this study, it was demonstrated that there was functional connectivity between other brain regions and $\mathrm{MrD}$ in the NCs and the patients with migraine. The normal connectivity pattern, such the positive associations with the bilateral basal ganglion nuclei, thalamus, insula, hippocampus, and medial frontal orbital cortex, and the negative associations with bilateral temporal lobes and middle frontal lobes, indicated that MrD was an important subcortical center. Previous report referred to it as a subcortical memory center. Interestingly, we found that $\mathrm{MrD}$ was also a subcortical pain center due to positive and negative associations with multiple brain 
Table 3 Comparison of functional connectivity of MrD among CM group, $\mathrm{MOH}+\mathrm{CM}$ group and EM group

\begin{tabular}{|c|c|c|c|c|c|c|c|}
\hline Groups & Brain region & k value & $P$ value & T value & $x$ & $y$ & z \\
\hline \multicolumn{8}{|l|}{ CM vs. EM } \\
\hline \multicolumn{8}{|l|}{$C M<E M$} \\
\hline Left & Cingulum_Mid_L & 10 & 0.000 & 3.9 & -9 & -24 & 39 \\
\hline Right & Cingulum_Mid_L & 42 & 0.000 & 5.0 & -6 & -27 & 39 \\
\hline \multicolumn{8}{|l|}{$\mathrm{CM}>\mathrm{EM}$} \\
\hline \multirow[t]{2}{*}{ Left } & Insula_R & 17 & 0.000 & 5.0 & 39 & 15 & 6 \\
\hline & Precentral_R & 12 & 0.000 & 4.7 & 66 & 3 & 21 \\
\hline \multicolumn{8}{|c|}{$\mathrm{MOH}+\mathrm{CM}$ vs.EM } \\
\hline \multicolumn{8}{|c|}{$\mathrm{MOH}+\mathrm{CM}<\mathrm{EM}$} \\
\hline \multirow[t]{2}{*}{ Left } & Frontal_Sup_Media_R & 15 & 0.000 & 4.4 & 12 & 60 & 24 \\
\hline & Supp_Motor_Area_L & 13 & 0.000 & 4.3 & -12 & -9 & 69 \\
\hline \multirow[t]{3}{*}{ Right } & Frontal_Sup_Media_L & 53 & 0.000 & 5.1 & -3 & 60 & 18 \\
\hline & Supp_Motor_Area_L & 24 & 0.000 & 4.1 & -9 & -12 & 66 \\
\hline & Precentral_L & 12 & 0.000 & 3.8 & -39 & 0 & 45 \\
\hline \multicolumn{8}{|c|}{$\mathrm{MOH}+\mathrm{CMvs} . \mathrm{CM}$} \\
\hline \multicolumn{8}{|c|}{$\mathrm{MOH}+\mathrm{CM}<\mathrm{CM}$} \\
\hline \multirow[t]{2}{*}{ Left } & Precentral_L & 19 & 0.000 & 4.9 & -45 & 0 & 51 \\
\hline & Frontal_Sup_Media_R & 11 & 0.000 & 4.4 & 9 & 60 & 18 \\
\hline \multirow[t]{3}{*}{ Right } & Frontal_Inf_Tri_L & 16 & 0.000 & 4.8 & -36 & 33 & 9 \\
\hline & Insula_L & 42 & 0.000 & 4.6 & -33 & 18 & 12 \\
\hline & Frontal_Sup_R & 10 & 0.000 & 3.8 & 21 & 63 & 12 \\
\hline \multicolumn{8}{|c|}{$\mathrm{MOH}+\mathrm{CM}>\mathrm{CM}$} \\
\hline Left & Temporal_Sup_L & 10 & 0.000 & 3.9 & -48 & -42 & 24 \\
\hline \multirow[t]{3}{*}{ Right } & Hippocampus_L & 13 & 0.000 & 4.8 & -27 & -18 & -18 \\
\hline & Cingulum_Mid_R & 14 & 0.000 & 4.1 & 3 & -27 & 33 \\
\hline & Thalamus_L & 10 & 0.000 & 3.7 & -15 & -18 & 9 \\
\hline
\end{tabular}

$R$ right hemisphere, $L$ left hemisphere, Ant anterior, Mid middle, Media medial, Sup superior, Inf inferior, Tri triangular part

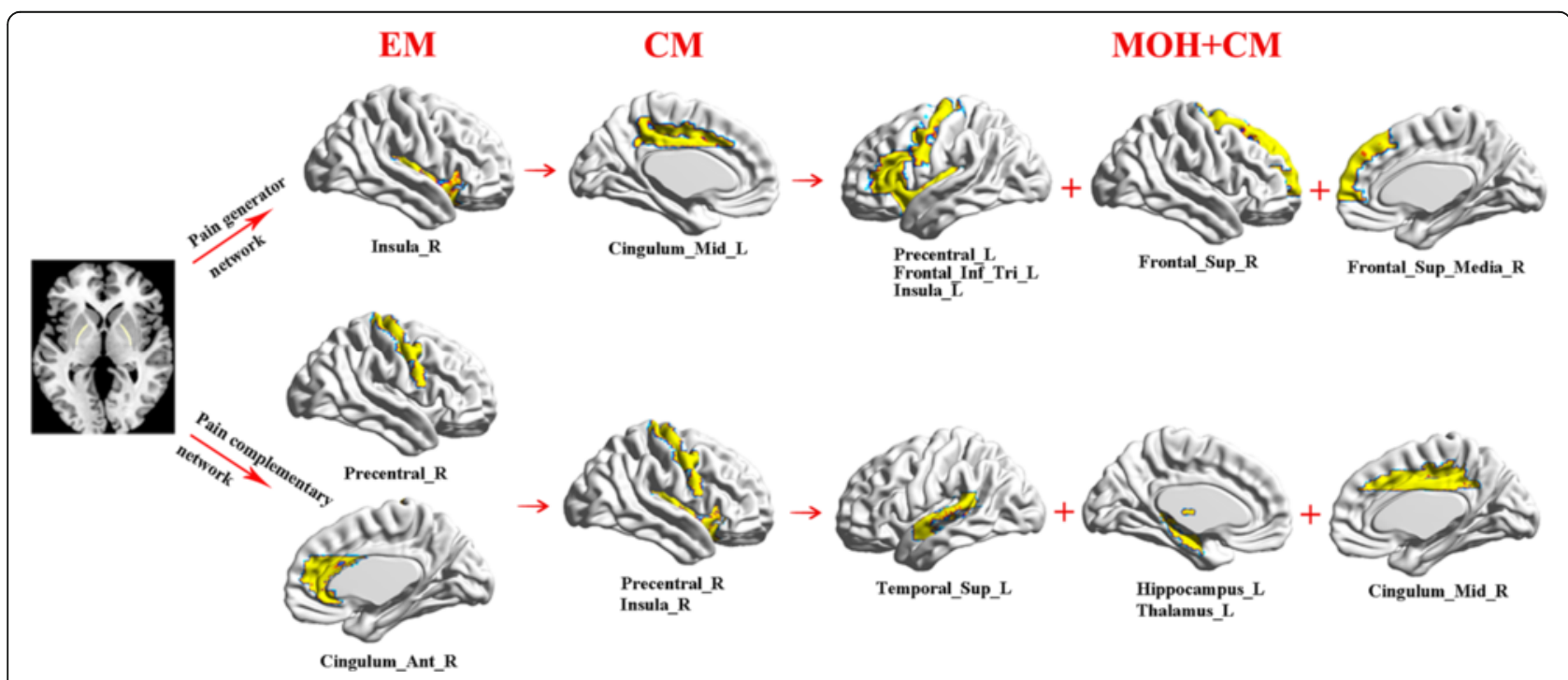

Fig. 4 Double neuromodulation network of MrD including three order pain generator network and three order pain complementary network 
regions in different subtypes of headache. In this study, only the positive connectivity was demonstrated in the EM group, and both the positive and negative connectivities were confirmed in the $\mathrm{CM}$ and $\mathrm{MOH}+\mathrm{CM}$ groups, which suggested that $\mathrm{MrD}$ demonstrated different pain modulation patterns in different subtypes of headache.

Between-groups analysis showed altered intrinsic functional connectivity in different subtypes of headache compared with NCs. The decreased functional connectivity of $\mathrm{MrD}$ was located in the right insula in the EM group, which was a component of the pain matrix. The insula seemed to play a leading role in the triggering of pain matrix network, and resulted in the subjective pain experience [29]. fMRI also demonstrated that the insula could process emotion and sensory-discriminative aspects of pain perception [30]. The impaired functional connectivity between $\mathrm{MrD}$ and the insula might disturb the balance of pain modulation of the insula in EM patients. Simultaneously, the functional connectivity was strengthened between the right precentral gyrus and the anterior cingulum cortex (ACC) and MrD. The increased connectivity could be understood as a positive feedback to maintain the concordance of the pain matrix network.

Compared with NCs, decreased functional connectivity was observed in the right cuneus and left middle cingulum in the CM group, which suggested that the cuneus and middle cingulum may be signature brain structures in chronic migraine, and may contribute to migraine chronicization. The cuneus is related to visual information processing [31], is responsible for the integration of the somatosensory information with other sensory stimuli and cognitive process [32], and could be activated with other pain-related brain regions [33]. The decreased functional connectivity between $\mathrm{MrD}$ and the cuneus may impair this integration, and facilitate migraine chronicization. The decreased functional connectivity between $\mathrm{MrD}$ and MCC was a new finding in CM patients compared previous studies [34-37] in which only the ACC was related to pain modulation, and the posterior cingulum cortex (PCC) was related to pain processing and cognitive impairment in migraineurs. The increased functional connectivity of $\mathrm{MrD}$ in the bilateral middle frontal gyrus, left hippocampus, and middle temporal gyrus indicated a dynamic compensation for the dysfunction of the painrelated brain regions in $\mathrm{CM}$ patients.

Only increased functional connectivity was confirmed in the left parahippocampus and right middle frontal and inferior temporal gyrus in the $\mathrm{MOH}+\mathrm{CM}$ group, which indicated that the dysfunction of $\mathrm{MrD}$ could not be detected. The reasons for this may be explained as follows: (1) The negative functional connectivity of MrD was activated, and the impaired MrD network was compensated and strengthened; (2) Medication overuse may inhibit the modulation function of $\mathrm{MrD}$ and provide some protection for $\mathrm{MrD}$. Therefore, the impairment of MrD connectivity was avoided. (3) The brain regions with increased functional connectivity may contribute to pain integration and protect the pain matrix.

Comparison of functional connectivity among different subtypes of headache demonstrated that $\mathrm{MrD}$ played a key role in migraine chronicization. The decreased functional connectivity of $\mathrm{MrD}$ could be detected from $\mathrm{EM}$ to $\mathrm{CM}$, from $\mathrm{EM}$ to $\mathrm{MOH}+\mathrm{CM}$, and from $\mathrm{CM}$ to $\mathrm{MOH}+\mathrm{CM}$. Additionally, the increased FM could also be detected from EM to $\mathrm{CM}$ and from $\mathrm{CM}$ to $\mathrm{MOH}+$ $\mathrm{CM}$. However, the increased functional connectivity was not revealed from $\mathrm{EM}$ to $\mathrm{MOH}+\mathrm{CM}$. The decreased connectivity pattern revealed the dysfunction of $\mathrm{MrD}$, which could explain the role of $\mathrm{MrD}$ in migraine chronicization. Therefore, MrD can be regarded as a subcortical pain center. The increased connectivity indicated that $\mathrm{MrD}$ could maintain the concordance of the pain matrix network.

On the basis of rs-fMRI findings, it can be speculated that $\mathrm{MrD}$ plays a double role in the neuromodulation of migraine (Fig. 4). One type of neuromodulation is the negative pain network, which includes three-order pain generating networks, and the functional connectivity is decreased among these networks. The first-order pain generating network is the right insula, which is the EM generator (EMG) and demonstrates decreased functional connectivity with $\mathrm{MrD}$. The second-order pain generating network is the left middle cingulate cortex (MCC), which is the CM generator (CMG) and also demonstrates decreased functional connectivity with $\mathrm{MrD}$. These findings also suggested that the left MCC played a key role in migraine chronicization. The last-order paingenerating network is the $\mathrm{MOH}+\mathrm{CM}$ generator (MOHG). This order pain network mainly included two brain regions: (1) the right superior frontal gyrus and medial superior frontal gyrus; (2) the left precentral gyrus, left pars triangularis of inferior frontal gyrus, and left insula.

The other neuromodulation is the positive pain network, which includes three-order pain complementary networks and the functional connectivity was strengthened among these networks. The first-order pain complementary network is located in the right precentral gyrus and right ACC, which may repair the dysfunction of $\mathrm{MrD}$ neuromodulation in EM patients. The secondorder pain complementary network is mainly revealed in the right precentral gyrus and right insula, which could compensate for the dysfunction of $\mathrm{MrD}$ neuromodulation and prevent migraine chronicization. The last-order pain complementary network is involved in the left superior temporal gyrus, left hippocampus, left thalamus, and right MCC. These brain regions may improve the state of the pain network in $\mathrm{MOH}+\mathrm{CM}$ patients. 
The double neuromodulation network of $\mathrm{MrD}$ indicated that the three-order pain generating network and the three-order pain complementary network were the important neuromodulation patterns of $\mathrm{MrD}$ in $\mathrm{mi}$ graines. The involved specific brain regions could be considered as target pain network biomarkers, and early-warning signals of neuromodulation in different subtypes of migraine.

There were some limitations in the present study. First, this study was a cross-sectional study, and the sample sizes of the EM and CM groups were relatively small. Future studies are needed to carry out longitudinal analysis to dynamically observe migraine chronicization and the real roles of MrD in this process. Second, task-based fMRIs should be performed to identify the key roles of $\mathrm{MrD}$ in pain modulation and transformation.

\section{Conclusions}

This study is the first to characterize the roles of $\mathrm{MrD}$ in the different subtypes of headache using rs-fMRI. The major findings are that the FC of $\mathrm{MrD}$ was demonstrated in the different subtypes of headache, and altered FC was revealed among different groups. These data indicated that $\mathrm{MrD}$ may play an important role in pain modulation and migraine chronicization, and the mechanism requires further investigation.

\section{Abbreviations}

CM: Chronic migraine; EM: Episodic migraine; $\mathrm{MOH}$ : Medication overuse headache; MrD: Marginal division; NC: Normal control

\section{Acknowledgments}

This work was supported by the National Natural Sciences Foundation of China (81371514), Special Financial Grant from the China Postdoctoral Science Foundation (2014 T70960) and the Foundation for Medical and health Sci \& Tech innovation Project of Sanya (2016YW37).

\section{Authors' contributions}

Category 1: (a) Conception and Design: LM; SYS; SYY. (b) Acquisition of Data: ZYC; MQL; SFL; XYC. (c) Analysis and Interpretation of Data: ZYC. Category 2: (a) Drafting the Article: ZYC. (b) Revising It for Intellectual Content: LM; SYY. All authors read and approved the final manuscript.

\section{Competing interests}

The authors declare that they have no competing interests.

\section{Author details}

'Department of Radiology, Chinese PLA General Hospital, Beijing 100853, China. ${ }^{2}$ Department of Neurology, Chinese PLA General Hospital, Beijing 100853, China. ${ }^{3}$ Institute of Cognitive Neuroscience, South China Normal University, Guangzhou 510631, China.

Received: 25 July 2016 Accepted: 22 September 2016 Published online: 26 September 2016

\section{References}

1. Shu SY, Penny GR, Peterson GM (1988) The 'marginal division': a new subdivision in the neostriatum of the rat. J Chem Neuroanat 1:147-163

2. Shu SY, Bao X, Li S, Niu D, Xu Z, Li Y (1999) A new subdivision of mammalian neostriatum with functional implications to learning and memory. J Neurosci Res 58:242-253
3. Shu SY, McGinty JF, Peterson GM (1990) High density of zinc-containing and dynorphin B- and substance P-immunoreactive terminals in the marginal division of the rat striatum. Brain Res Bull 24:201-205

4. Shu SY, Bao XM, Zhang X (1991) Ultrastructural characteristics of substance P-immunoreactive terminals in marginal division of rat striatum. Chin Med J (Engl) 104:887-896

5. Talley EM, Rosin DL, Lee A, Guyenet PG, Lynch KR (1996) Distribution of alpha 2A-adrenergic receptor-like immunoreactivity in the rat central nervous system. J Comp Neurol 372:111-134

6. Shu SY, Wu YM, Bao XM, Wen ZB, Huang FH, Li SX, Fu QZ, Ning Q (2002) A new area in the human brain associated with learning and memory: immunohistochemical and functional MRI analysis. Mol Psychiatry 7:1018-1022

7. Wu YM, Shu SY, Bao XM, Wen ZB, Huang FH, Yang WK, Liu YH (2002) Role of the marginal division of human neostriatum in working memory capacity for numbers received through hearing: a functional magnetic resonance imaging study. Di Yi Jun Yi Da Xue Xue Bao 22:1096-1098

8. Zhang ZQ, Shu SY, Liu SH, Guo ZY, Wu YM, Bao XM, Zheng JL, Ma HZ (2008) Activated brain areas during simple and complex mental calculationjA functional MRI study. Sheng Li Xue Bao 60:504-510

9. Chudler EH, Dong WK (1995) The role of the basal ganglia in nociception and pain. Pain 60:3-38

10. Chudler EH, Sugiyama K, Dong WK (1993) Nociceptive responses in the neostriatum and globus pallidus of the anesthetized rat. J Neurophysiol 69: 1890-1903

11. Liu XM, Shu SY, Zeng CC, Cai YF, Zhang KH, Wang CX, Fang J (2011) The role of substance $P$ in the marginal division of the neostriatum in learning and memory is mediated through the neurokinin 1 receptor in rats. Neurochem Res 36:1896-1902

12. Clark JW, Solomon GD, Senanayake PD, Gallagher C (1996) Substance P concentration and history of headache in relation to postlumbar puncture headache: towards prevention. J Neurol Neurosurg Psychiatry 60:681-683

13. Ma Y, Zhou C, Li G, Tian Y, Liu J, Yan L, Jiang Y, Tian S (2015) Effects on Spatial Cognition and Nociceptive Behavior Following Peripheral Nerve Injury in Rats with Lesion of the Striatal Marginal Division Induced by Kainic Acid. Neurochem Res 40:2357-2364

14. Wang C, Shu SY, Guo Z, Cai YF, Bao X, Zeng C, Wu B, Hu Z, Liu X (2011) Immunohistochemical localization of mu opioid receptor in the marginal division with comparison to patches in the neostriatum of the rat brain. J Biomed Sci 18:34

15. Zhang Z, Liu Y, Zhou B, Zheng J, Yao H, An N, Wang P, Guo Y, Dai H, Wang L, Shu S, Zhang $X$, Jiang T (2014) Altered functional connectivity of the marginal division in Alzheimer's disease. Curr Alzheimer Res 11:145-155

16. Chen Z, Li J, Liu M, Ma L (2013) Age-related changes in resting functional connectivity of the marginal division of the neostriatum in healthy adults. Nan Fang Yi Ke Da Xue Xue Bao 33:74-79

17. Chen Z, Li J, Liu M, Ma L (2013) Impact of gender on the marginal division of the neostriatum in health adults. Zhongguo Yi Xue Ke Xue Yuan Xue Bao 35:294-298

18. Chen Z, Ma L (2014) Right extremities pain caused by a malacia lesion in the left putamen:a resting functional magnetic resonance imaging of the marginal division of the human brain. Zhongguo Yi Xue Ke Xue Yuan Xue Bao 36:126-130

19. Headache Classification Committee of the International Headache Society (IHS) (2013) The International Classification of Headache Disorders, 3rd edition (beta version). Cephalalgia 33:629-808

20. Maier W, Buller R, Philipp M, Heuser I (1988) The Hamilton Anxiety Scale: reliability, validity and sensitivity to change in anxiety and depressive disorders. J Affect Disord 14:61-68

21. Hamilton M (1967) Development of a rating scale for primary depressive illness. Br J Soc Clin Psychol 6:278-296

22. Song XW, Dong ZY, Long XY, Li SF, Zuo XN, Zhu CZ, He Y, Yan CG, Zang YF (2011) REST: a toolkit for resting-state functional magnetic resonance imaging data processing. PLoS One 6:e25031

23. Scher Al, Gudmundsson LS, Sigurdsson S, Ghambaryan A, Aspelund T, Eiriksdottir G, van Buchem MA, Gudnason V, Launer L (2009) Migraine headache in middle age and late-life brain infarcts. JAMA 301:2563-2570

24. Yu S, Liu R, Zhao G, Yang X, Qiao X, Feng J, Fang Y, Cao X, He M, Steiner T (2012) The prevalence and burden of primary headaches in China: a population-based door-to-door survey. Headache 52:582-591

25. Lipton RB (2009) Tracing transformation: chronic migraine classification, progression, and epidemiology. Neurology 72:S3-S7 
26. Wiendels NJ, Knuistingh Neven A, Rosendaal FR, Spinhoven P, Zitman FG, Assendelft WJ, Ferrari MD (2006) Chronic frequent headache in the general population: prevalence and associated factors. Cephalalgia 26:1434-1442

27. Bingel U, Quante M, Knab R, Bromm B, Weiller C, Buchel C (2002)

Subcortical structures involved in pain processing: evidence from single-trial fMRI. Pain 99:313-321

28. Mainero C, Boshyan J, Hadjikhani N (2011) Altered functional magnetic resonance imaging resting-state connectivity in periaqueductal gray networks in migraine. Ann Neurol 70:838-845

29. Isnard J, Magnin M, Jung J, Mauguiere F, Garcia-Larrea L (2011) Does the insula tell our brain that we are in pain? Pain 152:946-951

30. Duerden EG, Albanese MC (2013) Localization of pain-related brain activation: a meta-analysis of neuroimaging data. Hum Brain Mapp 34:109-149

31. Calvert GA (2001) Crossmodal processing in the human brain: insights from functional neuroimaging studies. Cereb Cortex 11:1110-1123

32. Price DD (2000) Psychological and neural mechanisms of the affective dimension of pain. Science 288:1769-1772

33. de Leeuw R, Davis CE, Albuquerque R, Carlson CR, Andersen AH (2006) Brain activity during stimulation of the trigeminal nerve with noxious heat. Oral Surg Oral Med Oral Pathol Oral Radiol Endod 102:750-757

34. Kim JH, Kim S, Suh SI, Koh SB, Park KW, Oh K (2010) Interictal metabolic changes in episodic migraine: a voxel-based FDG-PET study. Cephalalgia 30:53-61

35. Valfre W, Rainero I, Bergui M, Pinessi L (2008) Voxel-based morphometry reveals gray matter abnormalities in migraine. Headache 48:109-117

36. Loggia ML, Edwards RR, Kim J, Vangel MG, Wasan AD, Gollub RL, Harris RE, Park K, Napadow V (2012) Disentangling linear and nonlinear brain responses to evoked deep tissue pain. Pain 153:2140-2151

37. Koppen H, Palm-Meinders I, Kruit M, Lim V, Nugroho A, Westhof I, Terwindt G, van Buchem M, Ferrari M, Hommel B (2011) The impact of a migraine attack and its after-effects on perceptual organization, attention, and working memory. Cephalalgia 31:1419-1427

\section{Submit your manuscript to a SpringerOpen ${ }^{\circ}$ journal and benefit from:}

- Convenient online submission

- Rigorous peer review

- Immediate publication on acceptance

- Open access: articles freely available online

- High visibility within the field

- Retaining the copyright to your article

Submit your next manuscript at $\gg$ springeropen.com 